\title{
Experimental Implementation of a Model-Based Inverse Filter to Attenuate Hysteresis in an Atomic Force Microscope
}

\author{
Andrew Hatch ${ }^{*}$, Ralph C. Smith* and Tathagata De ${ }^{\dagger}$ \\ ${ }^{*}$ Center for Research in Scientific Computation, North Carolina State University, Raleigh, NC 27695 \\ ${ }^{\dagger}$ Electrical Engineering Department, Iowa State University, Ames, IA 50011
}

\begin{abstract}
This paper addresses the development and experimental validation of a model-based, open loop control design for mitigating the frequency-dependent effects of hysteresis in an atomic force microscope (AFM). The models are based on homogenized energy relations which characterize the hysteretic constitutive behavior of the piezoceramic AFM stage. Approximate model inverses are then employed as filters to linearize transducer dynamics for control design. When experimentally implemented in open loop control designs, inverse compensation in this manner produces an approximately tenfold increase in tracking accuracy as compared with the unfiltered case.
\end{abstract}

\section{Introduction}

Crucial components in atomic force microscope (AFM) designs are the piezoceramic (PZT) drive mechanisms used to position the sample $[3,9]$. Whereas the specific designs vary, all employ the converse piezoelectric effect to provide input displacements and force in response to applied voltages or fields. This provides mechanisms which have nanometer-level setpoint accuracy but comes at the cost of the hysteresis and constitutive nonlinearities inherent to piezoelectric compounds.

To illustrate, consider the prototypical stage depicted in Figure 1(a) which employs stacked piezoceramic actuators to position the sample in the $x$ and $y$ directions. An additional mechanism provides transverse positioning capabilities. Nested minor loops collected at 0.1 $\mathrm{Hz}$ are plotted in Figure 1(b) and data collected at frequencies ranging from $0.279 \mathrm{~Hz}$ to $27.9 \mathrm{~Hz}$ is plotted in Figure 2 to illustrate the frequency-dependent nature of the hysteresis.

At low frequencies, the inherent hysteresis can be accommodated through PID or robust control designs $[1,2,4,5,7]$. However, at the higher frequencies required for applications ranging from real-time monitoring of biological processes - e.g., protein unfolding to comprehensive product diagnostics, increasing noiseto-data ratios and diminishing high-pass characteristics

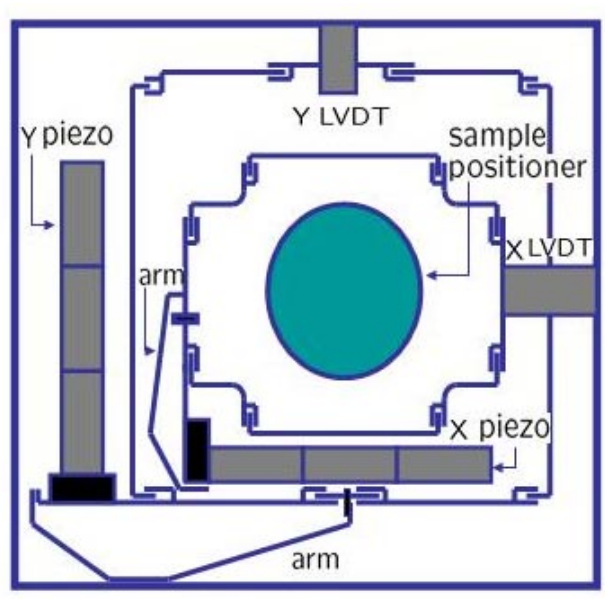

(a)

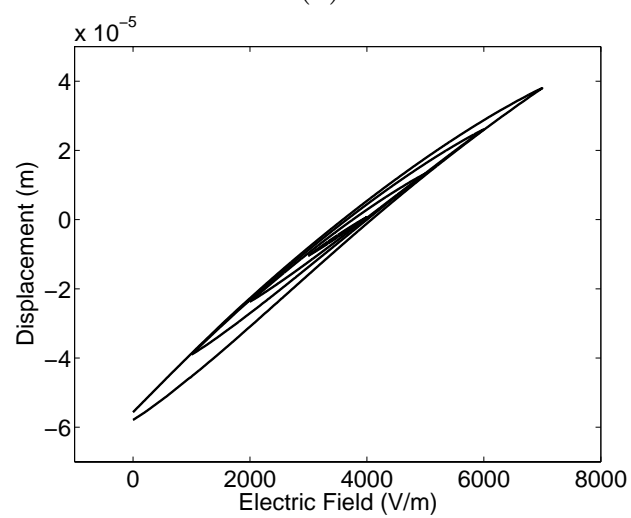

(b)

Figure 1. (a) PZT-based AFM stage. (b) Nested minor loops in AFM data collected at $0.1 \mathrm{~Hz}$.

of control filters preclude a sole reliance on feedback laws to eliminate hysteresis. This motivates the development of control designs that incorporate and approximately compensate for hysteresis through model inverses employed either in feedback or feedforward loops.

In this paper, we employ previously developed homogenized energy models to characterize the hysteretic relation between input fields and strains generated by 


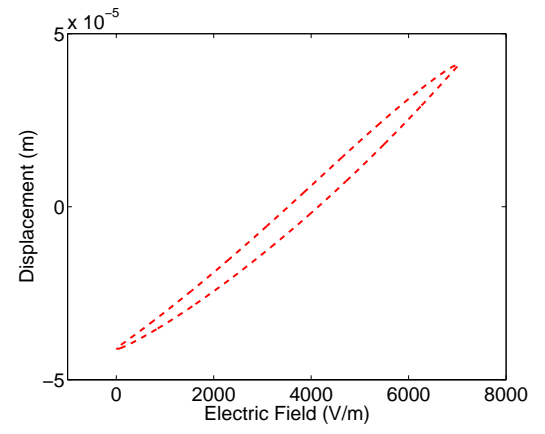

(a)

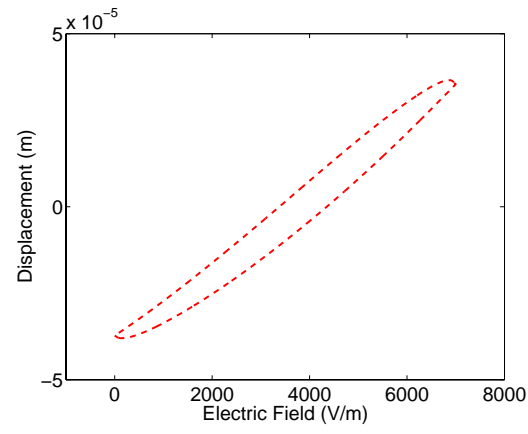

(b)

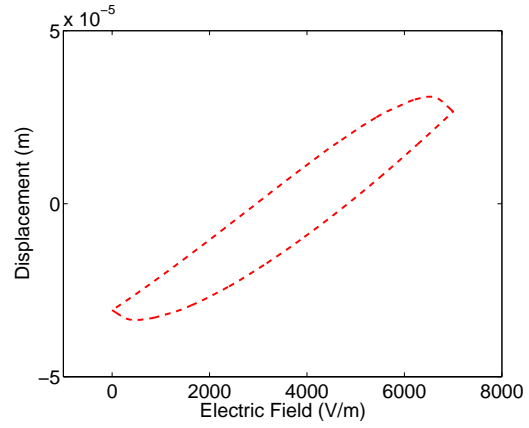

(c)

Figure 2. Frequency-dependent field-displacement behavior of an AFM. Sample rates of (a) $0.279 \mathrm{~Hz}$, (b) $5.58 \mathrm{~Hz}$, and (c) $27.9 \mathrm{~Hz}$.

the PZT rods. These constitutive laws are then employed to construct inverse models which serve to linearize the transducer response. When experimentally implemented in open loop control designs, it is illustrated that inverse compensation in this manner yields an approximately tenfold increase in accuracy over the unfiltered case when operating at $27.9 \mathrm{~Hz}$.

\section{Constitutive Relations}

To model the constitutive behavior of the piezoceramic stacked actuator, the stress-strain relation is assumed to be linear. However, the relation between the applied voltage $V$ (or the applied field $E$ ) and the polarization $P$ exhibits nonlinearities and hysteresis. The actuator is also assumed to be biased through poling so that the relation between $P$ and the strain $\varepsilon$ is linear for the considered operating conditions. To characterize the hysteretic $E-P$ behavior at the domain level, a Helmholtz energy relation was derived in [10] using statistical mechanics principles under the assumption that dipoles are either aligned with the field or diametrically opposed to it. This model is appropriate for a single crystal with uniform effective fields. To construct a macroscopic model for a polycrystalline material with variable effective fields, the coercive and effective field values are then assumed to be distributed as detailed in the latter half of this section.

Under fixed temperature conditions with no applied stress $\sigma$, it is illustrated in [10] that a first order approximation to the statistical mechanics-based Helmholtz energy is the piecewise quadratic relation

$$
\psi(P)= \begin{cases}\frac{1}{2} \eta\left(P+P_{R}\right)^{2} & P \leq-P_{I} \\ \frac{1}{2} \eta\left(P-P_{R}\right)^{2} & P \geq P_{I} \\ \frac{1}{2} \eta\left(P_{I}-P_{R}\right)\left(\frac{P^{2}}{P_{I}}-P_{R}\right) & |P|<P_{I} .\end{cases}
$$

As shown in Figure 3, $P_{I}$ is the positive inflection point and $P_{R}$ is the value of $P$ at which the positive local minimum of $\psi$ occurs. The parameter $\eta$ is the reciprocal of the slope of the $E$ - $P$ relation after switching occurs. This fact can be used to establish an initial parameter value for $\eta$ when modeling a specific data set.

In the case of no applied stresses $\sigma$, the Gibbs energy can be formulated as

$$
G(E, P)=\psi-E P
$$

where the second term represents the electrostatic energy due the applied field $E$. In order to include ferroelastic coupling, we utilize the extended Helmholtz relation

$$
\psi_{e}(P, \varepsilon)=\psi(P)+\frac{1}{2} Y^{P} \varepsilon^{2}-Y^{P} \gamma \varepsilon P .
$$

The Gibbs energy is then given by

$$
G(E, P, \varepsilon)=\psi(P)+\frac{1}{2} Y^{P} \varepsilon^{2}-Y^{P} \gamma \varepsilon P-E P-\sigma \varepsilon
$$
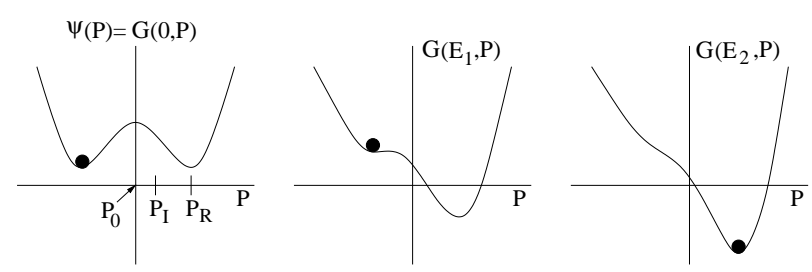

(a)
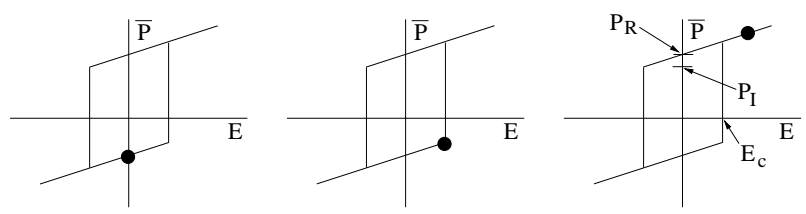

(b)

Figure 3. (a) Helmholtz energy $\psi$ and Gibbs energy $G$ for $\sigma=0$ and increasing fields $E$. (b) Dependence of the local polarization $\bar{P}$ on the field $E$ at the lattice level in the absence of thermal activation. 
where $\sigma \varepsilon$ incorporates the elastic energy. Note that $Y^{P}$ is the Young's modulus for a constant polarization and $\gamma$ is a ferroelastic coupling coefficient.

In the case of negligible thermal activation, the local average polarization $\bar{P}$ is determined from the necessary conditions

$$
\frac{\partial G}{\partial P}=0 \quad, \quad \frac{\partial^{2} G}{\partial P^{2}}>0 .
$$

Applying these conditions to (2) yields a piecewise linear $E-P$ characterization

$$
\left[\bar{P}\left(E, \varepsilon ; E_{c}, \xi\right)\right](t)=\left\{\begin{array}{l}
{\left[\bar{P}\left(E, \varepsilon ; E_{c}, \xi\right)\right](0)} \\
\frac{E}{\eta}-\frac{P_{R} \eta}{\eta-2 Y^{P} \gamma \varepsilon} \\
\frac{E}{\eta}+\frac{P_{R} \eta}{\eta-2 Y^{P} \gamma \varepsilon}
\end{array}\right.
$$

for the respective cases $\{\tau(t)=\emptyset\},\{\tau(t) \neq \emptyset$ and $\left.E(\max \tau(t))=-E_{c}\right\},\{\tau(t) \neq \emptyset$ and $E(\max \tau(t))=$ $\left.E_{c}\right\}$. The transition points $\tau$ are specified by

$$
\tau(t)=\left\{t \in\left(0, T_{f}\right] \mid E(t)=-E_{c} \text { or } E(t)=E_{c}\right\},
$$

and

$$
\left[\bar{P}\left(E, \varepsilon ; E_{c}, \xi\right)\right](0)=\left\{\begin{array}{l}
\frac{E}{\eta}-\frac{P_{R} \eta}{\eta-2 Y^{P} \gamma \varepsilon} \\
\xi \\
\frac{E}{\eta}+\frac{P_{R} \eta}{\eta-2 Y^{P} \gamma \varepsilon}
\end{array}\right.
$$

denotes the initial dipole orientation for respective initial fields $\left\{E(0) \leq-E_{c}\right\},\left\{-E_{c}<E(0)<E_{c}\right\}$ or $\left\{E(0) \geq E_{c}\right\}$.

However, if thermal activation is significant, dipoles can achieve the thermal energy required to switch in advance of the minimum Gibbs energy so the relative thermal and Gibbs energy must be balanced through Boltzmann principles. The probability density for achieving an energy level $G$ is then given by

$$
\mu(G)=C e^{-G V / k T}
$$

where $k$ is Boltzmann's constant, $V$ is a reference volume and $C$ is a constant that is selected so that when $\mu(G)$ is integrated over all possible dipole orientations, a probability of 1 is achieved. If we let $2 \sigma$ be the separation between possible polarization states around $P_{0}$, the probabilities of reaching a polarization state having sufficient energy to switch orientations are given by

$$
\begin{gathered}
r_{+-}=\frac{\int_{P_{0}-\sigma}^{P_{0}+\sigma} e^{-G(E, P) V / k T} d P}{\int_{P_{0}-\sigma}^{\infty} e^{-G(E, P) V / k T} d P} \\
r_{-+}=\frac{\int_{P_{0}-\sigma}^{P_{0}+\sigma} e^{-G(E, P) V / k T} d P}{\int_{-\infty}^{P_{0}+\sigma} e^{-G(E, P) V / k T} d P} .
\end{gathered}
$$

The likelihoods of reaching the required energy and thus of the dipoles switching from a positive to a negative orientation and conversely are then

$$
p_{+-}=\frac{1}{\tau} r_{+-} \quad, \quad p_{-+}=\frac{1}{\tau} r_{-+}
$$

where $\tau$ is the relaxation time. The fractions of dipoles in each orientation evolve according to the ordinary differential equations

$$
\begin{aligned}
& \frac{d x_{+}}{d t}=-p_{+-} x_{+}+p_{-+} x_{-} \\
& \frac{d x_{-}}{d t}=-p_{-+} x_{-}+p_{+-} x_{+} .
\end{aligned}
$$

The expected polarizations due to positively and negatively oriented dipoles are

$$
\left\langle P_{+}\right\rangle=\int_{P_{0}+\sigma}^{\infty} P \mu(G) d P,\left\langle P_{+}\right\rangle=\int_{-\infty}^{P_{0}-\sigma} P \mu(G) d P
$$

so that evaluation of $C$ yields

$$
\begin{array}{r}
\left\langle P_{+}\right\rangle=\frac{\int_{P_{0}+\sigma}^{\infty} P e^{-G(E, P, T) V / k T} d P}{\int_{P_{0}+\sigma}^{\infty} e^{-G(E, P, T) V / k T} d P} \\
\left\langle P_{-}\right\rangle=\frac{\int_{-\infty}^{P_{0}-\sigma} P e^{-G(E, P, T) V / k T} d P}{\int_{-\infty}^{P_{0}-\sigma} e^{-G(E, P, T) V / k T} d P} .
\end{array}
$$

For a single crystal with uniform effective field, the local average polarization is subsequently

$$
\bar{P}=x_{+}\left\langle P_{+}\right\rangle+x_{-}\left\langle P_{-}\right\rangle .
$$

In the manner detailed in [10], the evaluation of the integrals in (8) and (11) can be simplified through approximations employing the inflection points $\pm P_{I}$ rather than the unstable equilibrium $P_{0}$.

Both of the relations (6) and (13) are valid only for homogeneous single crystal materials with uniform effective fields. To account for nonuniformity and inhomogeneities in the materials, local coercive and effective fields are assumed to be manifestations of underlying distributions rather than constants. The macroscopic polarization model is then given by

$P(E)=\int_{0}^{\infty} \int_{-\infty}^{\infty} \bar{P}\left(E+E_{e}, E_{c}, \xi\right) \nu_{1}\left(E_{c}\right) \nu_{2}\left(E_{e}\right) d E_{e} d E_{c}$

where $\nu_{1}$ and $\nu_{2}$ are appropriate densities. Motivated by choices in the magnetics literature, $\nu_{1}$ and $\nu_{2}$ were respectively designated to be lognormal and normal densities in [10]. However, the fact that neither of these choices is based on energy considerations, motivates consideration of general densities.

Based on physical arguments, we may assume that the general densities decay to zero. Therefore, the double integral can be evaluated by truncating the domains and using composite Gaussian quadrature. In this case, the discretization of (14) gives

$$
P(E)=\sum_{i=1}^{N_{i}} \sum_{j=1}^{N_{j}} \bar{P}\left(E+E_{e_{j}}, E_{c_{i}}, \xi_{i}\right) \nu_{1}\left(E_{e_{j}}\right) \nu_{2}\left(E_{c_{i}}\right) v_{i} w_{j}
$$


where $E_{e_{j}}, E_{c_{i}}$ are the abscissas and $v_{i}, w_{j}$ are the weights. Here, $\nu_{1}\left(E_{e_{j}}\right)$ and $\nu_{2}\left(E_{c_{i}}\right)$ are parameter values to be determined. Details regarding the identification of $\nu_{1}$ and $\nu_{2}$ can be found in [6].

Finally, the equilibrium condition

$$
\frac{\partial G}{\partial \varepsilon}=0
$$

yields the elastic constitutive relation

$$
\sigma=Y^{P} \varepsilon-Y^{P} \gamma P
$$

This relation along with the nonlinear polarization relation (14) quantifies the constitutive behavior for the piezoceramic materials employed in the AFM.

\section{Model Inverse}

To construct a model inverse to be employed in subsequent control design, we exploit the approximate monotonicity of the $E-P$ relation to construct an algorithm in which the forward model is advanced until the prescribed polarization is crossed. Interpolation is then used to specify a final polarization value corresponding to the input field. This process is outlined Algorithm 1.

\section{Algorithm 1.}

for $k=2: N_{k}$

$$
\begin{aligned}
& \text { Specify } \Delta E \\
& \quad d P=P_{k}-P_{k-1} \\
& E_{t m p}=E_{k-1}, P_{t m p}=P_{k-1} \\
& \text { while } \operatorname{sgn}(d P) \cdot\left(P_{k}-P_{k-1}\right)>=0 \\
& \quad E_{t m p}=E_{t m p}+\Delta E \\
& \quad P_{t m p} \text { given by }(15) \\
& \text { end }
\end{aligned}
$$

$$
E_{k} \text { given by linear interpolation }
$$

end

\section{Stacked Actuator Model}

In addition to hysteresis, the dynamics of the actuator must be incorporated. We assume that the rod has cross-sectional area $A$, length $\ell$, density $\rho$ and Young's modulus $Y^{P}$. Let $c^{P}$ be the Kelvin-Voigt damping parameter and $\gamma$ be the piezoelectric coupling coefficient. In the present stage design, depicted in Figure 1(a), one end of the actuator is fixed, while the attachment at the other end can be modeled as a damped spring-mass system. For this end, let $M_{L}$ be the mass, $k_{L}$ be the stiffness, and $c_{L}$ be the damping coefficient. Force balancing along the actuator then yields the relation

$$
\rho A \frac{\partial^{2} u}{\partial t^{2}}=\frac{\partial \mathcal{N}}{\partial x}
$$

where the resultant $\mathcal{N}=\int_{A} \sigma d A$ is given by

$$
\mathcal{N}=Y^{P} A \frac{\partial u}{\partial x}+c^{P} A \frac{\partial^{2} u}{\partial x \partial t}-Y^{P} A \gamma P(E) .
$$

Note that $u$ denotes displacement in the longitudinal $x$ direction and that the relation strain $\varepsilon=\frac{\partial u}{\partial x}$ is used in obtaining (19). The boundary conditions are $u(t, 0)=0$ at the fixed end and

$$
\mathcal{N}(t, \ell)=-k_{L} u(t, \ell)-c_{L} \frac{\partial u}{\partial t}(t, \ell)-M_{L} \frac{\partial^{2} u}{\partial t^{2}}(t, \ell)
$$

at the moving end. Initial conditions are given by $u(0, x)=$ $\frac{\partial u}{\partial t}=0$. The polarization $P(E)$ is specified by (14).

An approximate solution to (18) is found by first deriving a weak form

$$
\begin{gathered}
\int_{0}^{\ell} \rho A \frac{\partial^{2} u}{\partial t^{2}} \psi d x+\int_{0}^{\ell}\left[Y^{P} A \frac{\partial u}{\partial x}+c^{P} A \frac{\partial^{2} u}{\partial x \partial t}\right] \frac{\partial \psi}{\partial x} d x \\
=-\int_{0}^{\ell} Y^{P} A \beta P(E) \frac{\partial \psi}{\partial x} d x
\end{gathered}
$$

and then discretizing in space with linear finite elements and in time with finite difference techniques. Details of the derivation of the weak form and the construction of the finite element and finite difference equations can be found in [9].

In order to determine the displacement of the rod at the end $x=\ell$, the rod model can be approximated by a lumped spring-mass model since the cross-section is small compared to the length and the electric field $E$ is approximately uniform throughout the actuator. $\mathrm{Nu}$ merical simulations and physical experiments demonstrate that the resulting ODE model is a good approximation to the PDE model for specifying tip displacement. The ODE model has the advantage of faster computational times compared to the discretized PDE.

\section{Model Validation}

To demonstrate the accuracy and efficiency of the hysteresis model, we consider the characterization of nested minor loops collected at $0.1 \mathrm{~Hz}$ as well as $E-P$ behavior at frequencies ranging from $0.279 \mathrm{~Hz}$ to $27.9 \mathrm{~Hz}$. In both cases, displacements were computed using the ODE model constructed using the stress relation (17) and hysteresis model (14) with general densities $\nu_{1}$ and $\nu_{2}$ identified using the techniques detailed in $[6,8]$.

Figure 4 demonstrates the characterization of frequency dependent dynamics. The latter involves the quantification of both thermal relaxation and inertial effects as illustrated by the change in sign of the slope $\frac{\partial P}{\partial E}$ following field reversal. Further details demonstrating properties of the model for characterizing hysteresis in various PZT compounds can be found in $[6,8,10]$. 


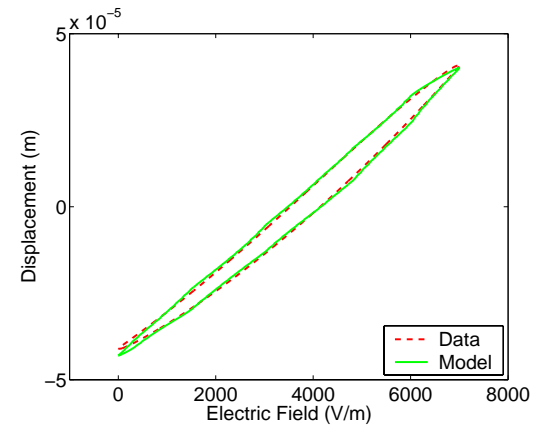

(a)

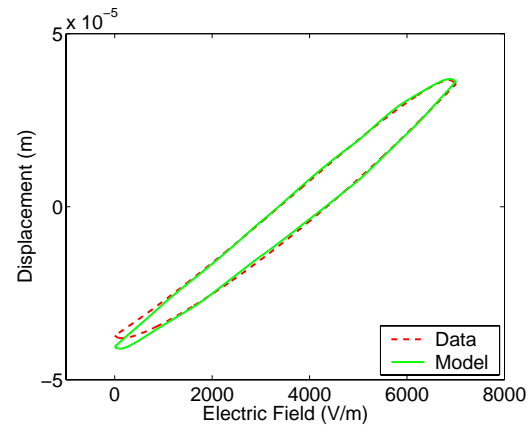

(b)

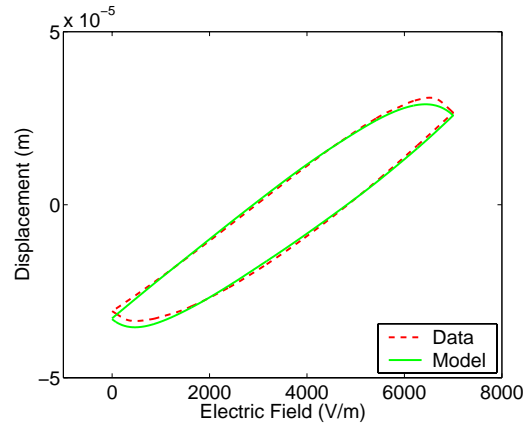

(c)

Figure 4. Characterization of AFM field-displacement behavior with sample rates of (a) $0.279 \mathrm{~Hz}$, (b) $5.58 \mathrm{~Hz}$ and (c) $27.9 \mathrm{~Hz}$.

\section{Open Loop Control Implementation}

To illustrate the effect of filters employing the inverse model from Section 3 on open loop tracking performance, we summarize experiments conducted at $0.279 \mathrm{~Hz}$ and $27.9 \mathrm{~Hz}$. In both cases, the specified trajectory to be tracked consisted of a triangle wave ranging from 0 to $7000 \mathrm{~V} / \mathrm{m}$. To construct the model-based inverse filter, the specified trajectory was input to the inverse model and the resulting field was applied to the experimental device. To provide a metric for comparison, a second predicted input field for each case was determined by a linear scaling of the desired output displacement. The scaling factors were derived from manufacturer specifications for the actuator stroke when the field is increased throughout its range.

The specified and achieved trajectories, errors, and hysteresis plots for the two frequencies are plotted in Figures 5 and 6 . At $0.279 \mathrm{~Hz}$, the filtered design provides only marginally improved accuracy due to the low degree of hysteresis and constitutive nonlinearities. For the more hysteretic response at $27.9 \mathrm{~Hz}$, however, the inverse filter provides a significant increase in accuracy and yields errors that are approximately a factor of 10 smaller than the unfiltered but scaled case. This illustrates the advantage of incorporating the frequencydependent model inverse in the control design.

We note that the primary source of errors in the filtered design is variability between experiments as illustrated by the variation in the hysteresis plots at each frequency. This is hypothesized to be due to variations in the true applied voltage and illustrates one reason feedback is necessary in final control designs.

\section{Concluding Remarks}

The modeling framework employed in this paper provides the capability for characterizing the frequencydependent hysteresis inherent to the piezoceramic drive mechanisms in atomic force microscopes. It is also ame-
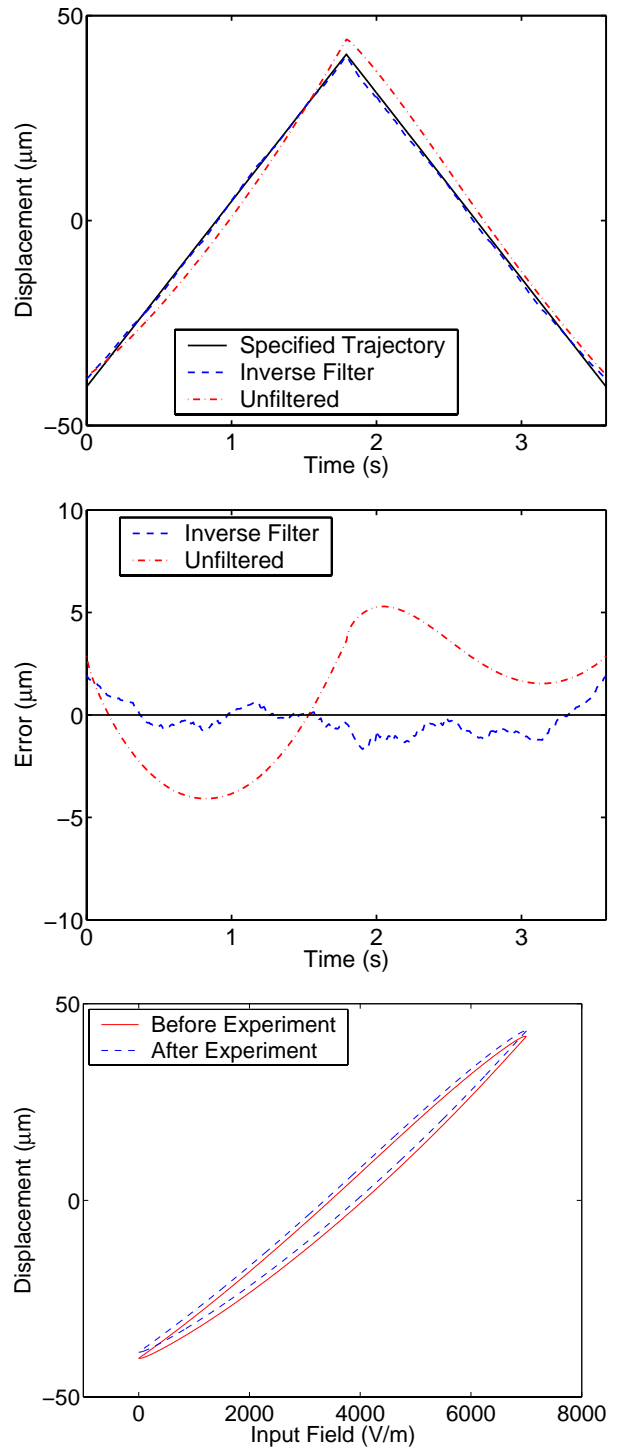

Figure 5. Tracking performance utilizing the modelbased filter and without a filter at $0.279 \mathrm{~Hz}$. 

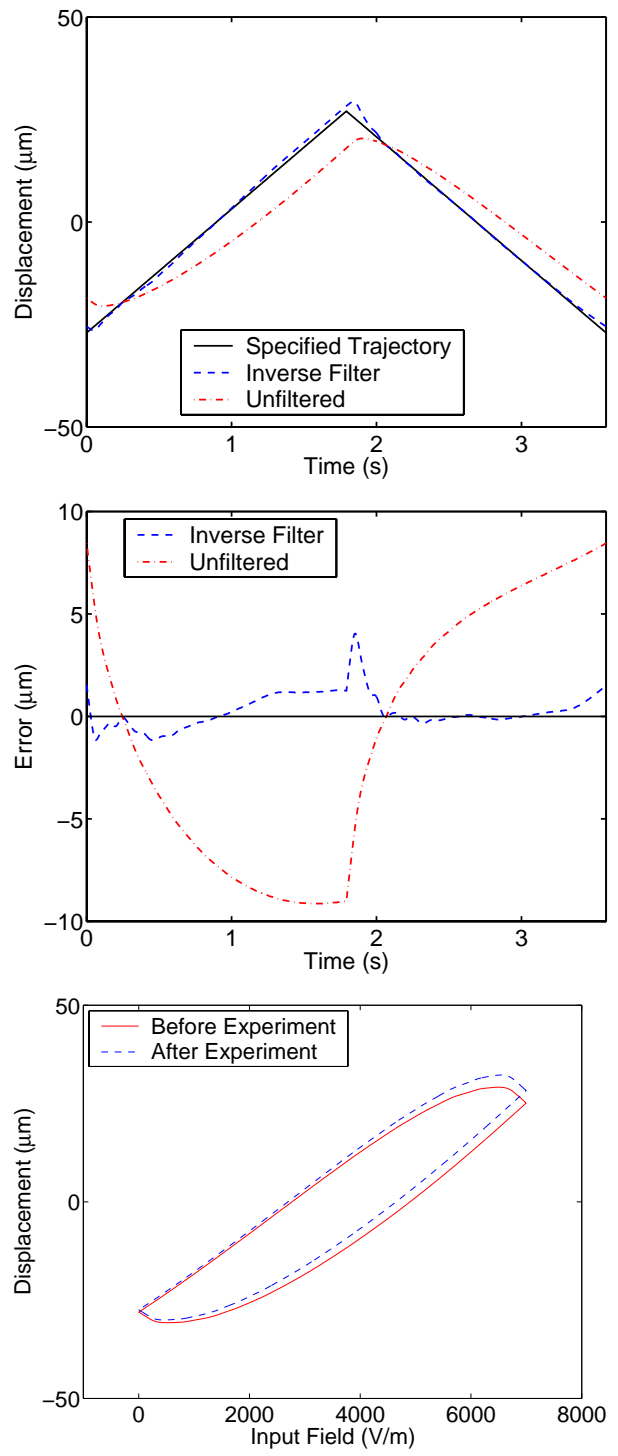

Figure 6. Tracking performance utilizing the modelbased filter and without a filter at $27.9 \mathrm{~Hz}$

nable to inversion and hence can be employed to construct inverse filters which linearize the transducer dynamics as a prelude to linear control designs. Experimental implementation of an open loop control design employing the filter illustrates that tracking errors are reduced by a factor of approximately ten as compared with the unfiltered case. Present investigations are focused on the design and experimental implementation of closed loop feedback algorithms which employ the inverse filters.

\section{Acknowledgements}

This research was supported by the NSF grants CMS0099764 and CMS-0201560, the Air Force Office of Scientific Research under the grant AFOSR-F49620-01-1-
0107, and the DARPA MOSAIC Program through the grant 1000-G-CF980.

\section{References}

[1] D. Croft, G. Shed and S. Devasia, "Creep, hysteresis, and vibration compensation for piezoactuators: Atomic force microscopy application," Journal of Dynamic Systems, Measurement, and Control, 23, pp. 35-43, 2001.

[2] A. Daniele, S. Salapaka, M.V. Salapaka and M. Dahleh, "Piezoelectric scanners for atomic force microscopes: Design of lateral sensors, identification and control," Proceedings of the America Control Conference, San Diego, CA, pp. 253-257, 1999.

[3] P.K. Hansma, V.B. Elings, O. Marti and C.E. Bracker, "Scanning tunneling microscopy and atomic force microscopy: Application to biology and technology," Science, 242, pp. 209-242, 1988.

[4] J.M. Nealis and R.C. Smith, " $\mathcal{H}_{\infty}$ Control Design for a Magnetostrictive Transducer," Proc. 42nd IEEE Conf. Dec. and Control, Maui, HA, pp. 18011806, 2003.

[5] S. Salapaka, A. Sebastian, J.P. Cleveland and M.V. Salapaka, "High bandwidth nano-positioner: A robust control approach," Review of Scientific Instruments, 73(9), pp. 3232-3241, 2002.

[6] R.C. Smith and A. Hatch, "Parameter estimation techniques for nonlinear hysteresis models," Proceedings of the SPIE, Smart Structures and Materials, to appear.

[7] R.C. Smith, A. Hatch and T. De, "Model Development for Piezoceramic Nanopositioners," Proc. 42nd IEEE Conf. Dec. and Control, Maui, HA, pp. 2638-2643, 2003.

[8] R.C. Smith, A. Hatch, B. Mukherjee and S. Liu, "A homogenized energy model for hysteresis in ferroelectric materials: General density formulation," Journal of Intelligent Material Systems and Structures, to appear.

[9] R.C. Smith and M. Salapaka, "Model development for the positioning mechanisms in an atomic force microscope," International Series of Numerical Mathematics, Vol 143, pp. 249-269, 2002.

[10] R.C. Smith, S. Seelecke, Z. Ounaies and J. Smith, "A free energy model for hysteresis in ferroelectric materials," Journal of Intelligent Material Systems and Structures, 14(11), pp. 719-739, 2003. 